Supplement of Biogeosciences Discuss., 12, 12713-12759, 2015

http://www.biogeosciences-discuss.net/12/12713/2015/

doi:10.5194/bgd-12-12713-2015-supplement

(C) Author(s) 2015. CC Attribution 3.0 License.

(c) (1)

Supplement of

\title{
Ocean dynamic processes causing spatially heterogeneous distribution of sedimentary caesium-137 massively released from the Fukushima Dai-ichi Nuclear Power Plant
}

\section{H. Higashi et al.}

Correspondence to: H. Higashi (higashi@nies.go.jp)

The copyright of individual parts of the supplement might differ from the CC-BY 3.0 licence. 
1 Table S1. Evaluation of our model performance for seawater-surface ${ }^{137} \mathrm{Cs}$ at 10 TEPCO 2 (2011) stations shown in Fig. 4j. Values of $N$ indicate number of sampling data, except for 3 non-detected. Statistical indexes $F A n$ and $F B$ are described in Appendix A. $F B$ in the total 4 row indicates an all-station average when weights W-1 and W-2 are half those of the other 5 stations, because $\mathrm{W}-1$ and $\mathrm{W}-2$ are within same simulation grid.

6

\begin{tabular}{cccccc}
\hline Stn & $N$ & $F B$ & $F A 5(\%)$ & $F A 3(\%)$ & $F A 2(\%)$ \\
\hline W-1 & 188 & -1.42 & 89.4 & 75.0 & 48.4 \\
W-2 & 192 & -1.19 & 92.7 & 86.5 & 67.7 \\
W-3 & 125 & -0.66 & 95.2 & 69.6 & 48.0 \\
W-4 & 86 & -0.98 & 89.5 & 79.1 & 48.8 \\
W-5 & 18 & -0.33 & 83.3 & 66.7 & 50.0 \\
W-6 & 21 & -0.09 & 90.5 & 76.2 & 57.1 \\
W-7 & 23 & -0.14 & 100.0 & 87.0 & 56.5 \\
W-8 & 22 & -0.89 & 81.8 & 59.1 & 50.0 \\
W-9 & 22 & -1.51 & 50.0 & 27.3 & 13.6 \\
W-10 & 19 & -1.34 & 57.9 & 26.3 & 15.8 \\
\hline Total & 716 & -0.81 & 89.2 & 74.6 & 52.2 \\
\hline
\end{tabular}

7

8 
1 Table S2. Evaluation of our model performance for sediment-surface ${ }^{137} \mathrm{Cs}$ at $30 \mathrm{MEXT}$ 2 (2011) stations in Fig. 2b. Values of $N$ indicate number of sampling data. Statistical indexes $3 F A n$ and $F B$ are described in Appendix A. $F B$ in the total row indicates an all-station average.

4

\begin{tabular}{|c|c|c|c|c|c|}
\hline Stn. & $N$ & $F B$ & $F A 5(\%)$ & $F A 3(\%)$ & $F A 2(\%)$ \\
\hline A1 & 9 & 1.26 & 44.4 & 33.3 & 22.2 \\
\hline A3 & 3 & 0.16 & 100.0 & 100.0 & 66.7 \\
\hline a1 & 3 & 1.28 & 66.7 & 0.0 & 0.0 \\
\hline B1 & 9 & 1.90 & 0.0 & 0.0 & 0.0 \\
\hline B3 & 3 & -0.60 & 100.0 & 100.0 & 66.7 \\
\hline $\mathrm{C} 1$ & 9 & 1.94 & 0.0 & 0.0 & 0.0 \\
\hline $\mathrm{C} 3$ & 3 & -0.68 & 100.0 & 100.0 & 66.7 \\
\hline D1 & 9 & -0.82 & 77.8 & 66.7 & 66.7 \\
\hline D3 & 3 & -0.27 & 100.0 & 100.0 & 100.0 \\
\hline E1 & 9 & -0.68 & 88.9 & 77.8 & 22.2 \\
\hline E3 & 3 & -0.45 & 100.0 & 100.0 & 100.0 \\
\hline E5 & 3 & 0.56 & 100.0 & 66.7 & 66.7 \\
\hline $\mathrm{F} 1$ & 9 & -0.26 & 88.9 & 77.8 & 66.7 \\
\hline F3 & 3 & -0.30 & 100.0 & 100.0 & 100.0 \\
\hline G0 & 3 & 0.09 & 100.0 & 100.0 & 100.0 \\
\hline G1 & 9 & 0.41 & 100.0 & 100.0 & 66.7 \\
\hline G3 & 3 & 0.58 & 100.0 & 100.0 & 33.3 \\
\hline G4 & 3 & -0.60 & 100.0 & 100.0 & 33.3 \\
\hline H1 & 9 & 0.74 & 100.0 & 77.8 & 44.4 \\
\hline H3 & 3 & 0.24 & 100.0 & 100.0 & 100.0 \\
\hline I0 & 3 & 0.93 & 100.0 & 66.7 & 0.0 \\
\hline I1 & 9 & 1.07 & 55.6 & 44.4 & 33.3 \\
\hline $\mathrm{I} 3$ & 3 & 0.54 & 100.0 & 100.0 & 66.7 \\
\hline $\mathrm{J} 1$ & 9 & 0.83 & 66.7 & 44.4 & 11.1 \\
\hline $\mathrm{J} 2$ & 3 & 0.35 & 100.0 & 100.0 & 66.7 \\
\hline $\mathrm{J} 3$ & 3 & -1.47 & 0.0 & 0.0 & 0.0 \\
\hline K1 & 9 & 1.23 & 66.7 & 33.3 & 11.1 \\
\hline $\mathrm{K} 2$ & 3 & 1.03 & 100.0 & 66.7 & 0.0 \\
\hline L1 & 9 & 0.56 & 88.9 & 77.8 & 55.6 \\
\hline L3 & 3 & -0.96 & 33.3 & 33.3 & 33.3 \\
\hline Total & 162 & 0.29 & 72.8 & 61.7 & 40.7 \\
\hline
\end{tabular}

5 
1 Table S3. Evaluation of our model performance for sediment-surface ${ }^{137} \mathrm{Cs}$ at 25 TEPCO 2 sampling stations in Fig. 2c. Values of $N$ indicate number of sampling data. Statistical indexes $3 F A n$ and $F B$ are described in Appendix A. $F B$ in the total row indicates an all-station average.

4

\begin{tabular}{|c|c|c|c|c|c|}
\hline Stn. & $N$ & $F B$ & $F A 5(\%)$ & $F A 3(\%)$ & $F A 2(\%)$ \\
\hline 24 & 5 & 1.27 & 80.0 & 40.0 & 20.0 \\
\hline 23 & 5 & 0.50 & 60.0 & 40.0 & 40.0 \\
\hline 22 & 5 & -1.83 & 60.0 & 60.0 & 40.0 \\
\hline 15 & 5 & 1.26 & 60.0 & 0.0 & 0.0 \\
\hline 12 & 9 & 1.68 & 44.4 & 22.2 & 11.1 \\
\hline 11 & 5 & 0.16 & 100.0 & 100.0 & 60.0 \\
\hline 1 & 5 & -0.81 & 80.0 & 40.0 & 20.0 \\
\hline 2 & 5 & -0.08 & 100.0 & 60.0 & 20.0 \\
\hline 3 & 5 & 0.68 & 100.0 & 100.0 & 60.0 \\
\hline 16 & 5 & 0.42 & 100.0 & 100.0 & 80.0 \\
\hline 6 & 5 & 1.81 & 0.0 & 0.0 & 0.0 \\
\hline 7 & 5 & 1.07 & 80.0 & 20.0 & 0.0 \\
\hline 5 & 5 & 1.90 & 0.0 & 0.0 & 0.0 \\
\hline 8 & 5 & 0.75 & 80.0 & 80.0 & 40.0 \\
\hline 9 & 5 & 0.73 & 60.0 & 60.0 & 20.0 \\
\hline 10 & 5 & 0.27 & 100.0 & 100.0 & 80.0 \\
\hline 13 & 9 & 1.13 & 88.9 & 88.9 & 55.6 \\
\hline 14 & 5 & 0.77 & 100.0 & 80.0 & 40.0 \\
\hline 4 & 5 & 0.75 & 100.0 & 80.0 & 0.0 \\
\hline 17 & 5 & 1.30 & 40.0 & 20.0 & 0.0 \\
\hline 20 & 5 & 1.09 & 100.0 & 0.0 & 0.0 \\
\hline 29 & 5 & 1.12 & 60.0 & 40.0 & 20.0 \\
\hline 21 & 5 & 0.77 & 100.0 & 100.0 & 20.0 \\
\hline 19 & 5 & 0.26 & 100.0 & 100.0 & 80.0 \\
\hline 18 & 5 & 0.87 & 100.0 & 60.0 & 40.0 \\
\hline Total & 134 & 0.71 & 75.2 & 55.6 & 30.1 \\
\hline
\end{tabular}

5 
1 Table S4. Evaluation of our model performance for vertical ${ }^{137}$ Cs activity profile in sediment 2 at (a) eight Otosaka and Kobayashi (2013) stations in Fig. 2c, and (b) four Otosaka and Kato 3 (2014) stations in Fig. 2b. Values of $N$ indicate number of sampling data. Values of $N$ indicate 4 number of sampling data. Statistical indexes $F A n$ and $F B$ are described in Appendix A. 5 Values of $F B$ in the total rows indicate all-layer averages.

6

7 (a) Nearshore region (Otosaka and Kobayashi, 2013)

\begin{tabular}{cccccc}
\hline Layer & $N$ & $F B$ & $F A 5(\%)$ & $F A 3(\%)$ & $F A 2(\%)$ \\
\hline $0-3 \mathrm{~cm}$ & 20 & 0.72 & 95.0 & 55.0 & 35.0 \\
$3-10 \mathrm{~cm}$ & 17 & 0.39 & 94.1 & 70.6 & 47.1 \\
\hline Total & 37 & 0.60 & 94.6 & 62.2 & 40.5 \\
\hline
\end{tabular}

8

9 (b) Offshore region (Otosaka and Kato, 2014)

\begin{tabular}{cccccc}
\hline Layer & $N$ & $F B$ & $F A 5(\%)$ & FA3 (\%) & FA2 (\%) \\
\hline $0-1 \mathrm{~cm}$ & 4 & -0.56 & 100.0 & 100.0 & 75.0 \\
$1-2 \mathrm{~cm}$ & 4 & -0.30 & 100.0 & 100.0 & 75.0 \\
$2-3 \mathrm{~cm}$ & 4 & 0.15 & 75.0 & 75.0 & 50.0 \\
$3-4 \mathrm{~cm}$ & 4 & 0.76 & 100.0 & 100.0 & 50.0 \\
$4-5 \mathrm{~cm}$ & 4 & 1.50 & 50.0 & 0.0 & 0.0 \\
$5-6 \mathrm{~cm}$ & 3 & 1.40 & 33.3 & 0.0 & 0.0 \\
$6-7 \mathrm{~cm}$ & 2 & 1.50 & 50.0 & 0.0 & 0.0 \\
$7-8 \mathrm{~cm}$ & 2 & 1.64 & 50.0 & 0.0 & 0.0 \\
$9-10 \mathrm{~cm}$ & 2 & 1.66 & 0.0 & 0.0 & 0.0 \\
\hline Total & 29 & 0.86 & 69.0 & 51.7 & 34.5 \\
\hline
\end{tabular}

10 


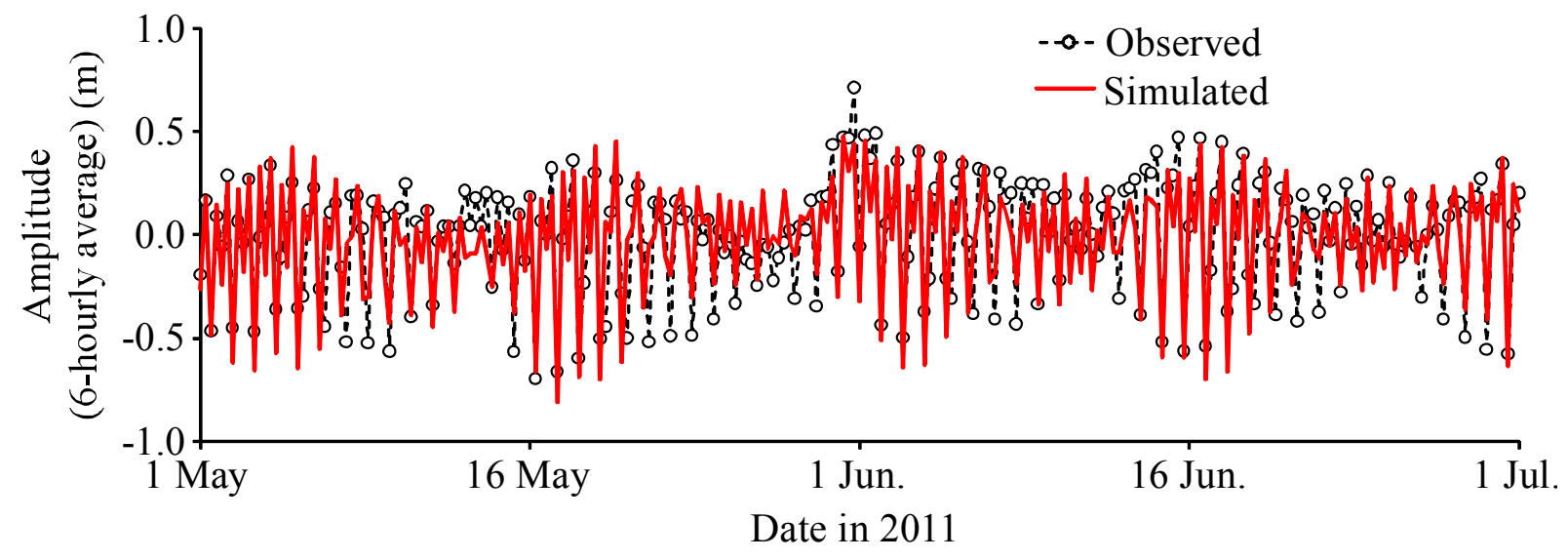

2

3 Figure S1. Comparison between observed and simulated tidal amplitude (6-hour average) at 4 Choshi Fishing Port $\left(140.87^{\circ} \mathrm{E}, 35.75^{\circ} \mathrm{N}\right)$. Observations were provided by JMA. 


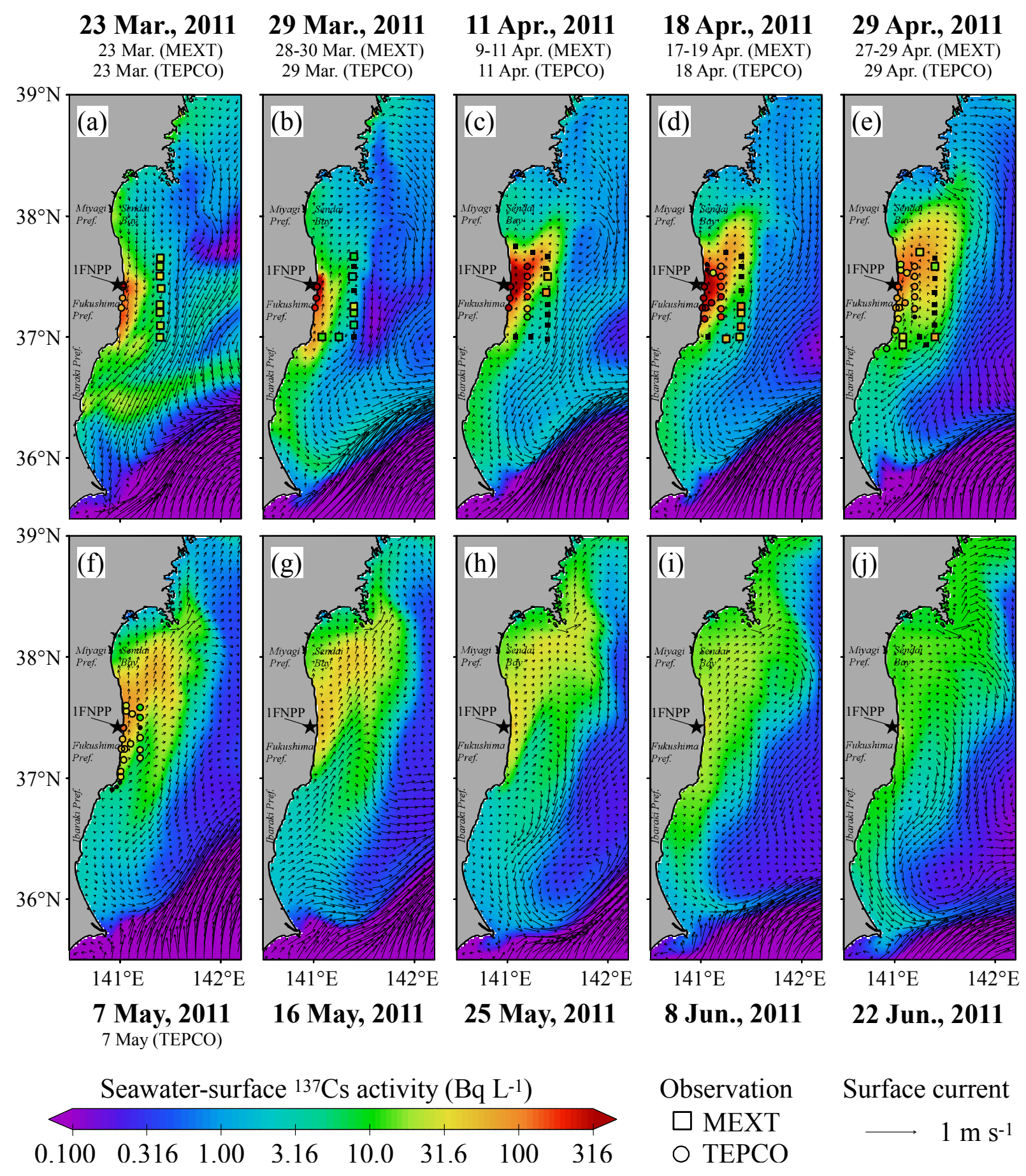

3 Figure S2. Spatial distributions of observed (colour plots of circles and squares) and simulated 4 (colour shading) seawater-surface ${ }^{137}$ Cs. Dates of simulated/observed results are denoted by 5 bold/normal characters. Line contours show water depth (m) according to JTOPO30 (MIRC). 6 Black star indicates location of 1FNPP. Observations are from MEXT (2011) and TEPCO 7 (2011). Observations of MEXT (2011) (f-j) and TEPCO (2011) $(g-j)$ were omitted because 8 most indicated non-detected. 